\title{
Trans-sphenoidal surgery for microprolactinoma: an acceptable alternative to dopamine agonists?
}

\author{
Helen E Turner, Christopher B T Adams ${ }^{1}$ and John A H Wass \\ Departments of Endocrinology and ${ }^{1}$ Neurosurgery, Radcliffe Infirmary, Woodstock Road, Oxford OX2 6HE, UK \\ (Correspondence should be addressed to J A H Wass)
}

\begin{abstract}
Aims: Reported cure rates following trans-sphenoidal surgery for microprolactinoma are variable and recurrence rates in some series are high. We wished to examine the cure rate of trans-sphenoidal surgery for microprolactinoma, and to assess the long-term complications and recurrence rate.

Design: A retrospective review of the outcome of trans-sphenoidal surgery for microprolactinoma, performed by a single neurosurgeon at a tertiary referral centre between 1976 and 1997.

Patients: All thirty-two patients operated on for microprolactinoma were female, with a mean age of 31 years (range 16-49). Indications for surgery were intolerance of dopamine agonists in ten (31\%), resistance in six $(19 \%)$ and resistance and intolerance in four $(12.5 \%)$. Two patients were from countries where dopamine agonists were unavailable.

Results: The mean pre-operative prolactin level was $2933 \mathrm{mU} / \mathrm{l}$ (range 1125-6000). All but 1 had amenorrhoea or oligomenorrhoea, with galactorrhoea in $15(46.9 \%)$. Twenty-five (78\%) were cured by trans-sphenoidal surgery, as judged by a post-operative serum prolactin in the normal range. During a mean follow-up of 70 months (range 2 months to 16 years) there was one recurrence at 12 years. Post-operatively, one patient became LH deficient, two patients became cortisol deficient and two became TSH deficient. Out of 21 patients tested for post-operative growth hormone deficiency, 6 (28.6\%) were deficient. Five patients developed post-operative diabetes insipidus which persisted for greater than 6 months. There were no other complications of surgery. The estimated cost of uncomplicated trans-sphenoidal surgery, and follow-up over 10 years, was similar to that of dopamine agonist therapy.

Conclusion: In patients with hyperprolactinaemia due to a pituitary microprolactinoma, transsphenoidal surgery by an experienced pituitary surgeon should be considered as a potentially curative procedure. The cost of treatment over a 10 year period is similar in uncomplicated cases to long-term dopamine agonist therapy.
\end{abstract}

European Journal of Endocrinology 140 43-47

\section{Introduction}

Dopamine agonists have been recognised as medical treatment for prolactinomas for over two decades $(1,2)$. A review of more than 400 patients with hyperprolactinaemia who were treated with bromocriptine found that $80-90 \%$ developed normal prolactin levels or a return of menses (3). Cabergoline, a long-acting dopamine agonist is tolerated in some patients who are bromocriptine intolerant (4) and may be more efficacious (5). Cabergoline was shown to reduce prolactin levels in 114/127 (90\%) hyperprolactinaemic patients, of whom 71 had microprolactinomas (6). Twenty-nine patients (23\%) described adverse effects. Cabergoline has also been shown to be a successful treatment for patients with microprolactinomas that are bromocriptine and quinagolide resistant, defined as absent or poor response (normalisation of prolactin and or tumour mass shrinkage) to $15 \mathrm{mg}$ bromocriptine for at least 3 months (7). However, dopamine agonists are not always effective, may have side-effects limiting their use, and the better tolerated ones (such as cabergoline) as yet lack adequate safety profiles to allow licensing in pregnancy.

Trans-sphenoidal surgery is currently seen as secondline treatment for the majority of patients with microprolactinomas, although this is a controversial issue (8). Most patients will be treated with dopamine agonists as initial therapy, with surgery reserved for those who either fail to respond or who cannot tolerate treatment. Variable cure rates judged by normalisation of prolactin levels following trans-sphenoidal surgery for microprolactinomas have been reported: $85 \%$ (9), $67 \%$ (10), $75 \%$ (11), $88 \%$ (12) and 70\% (13). Recurrence rates following trans-sphenoidal surgery for microprolactinomas have been reported as high as $50 \%$ of those with initially normal prolactin levels following surgery (9). Other published series have shown lower but still significant recurrence rates: $12 \%$ (14), $21 \%$ (12) and $17.4 \%$ (3). 


\section{Method and patients}

Thirty-six patients were identified from the database of endocrine patients as having trans-sphenoidal surgery for microprolactinomas between 1976 and 1997. Four patients were excluded, two because of insufficient data, and two because they were operated on elsewhere. The patients were all female, with a mean age of 31 years (range 16 to 49 years). All operations were performed by a single surgeon (CB TA). The mean duration of follow-up was 5.8 years (range 2 months to 16 years, median 4.5 years). A retrospective analysis of the data was performed.

Pituitary microadenomas were diagnosed on the basis of sustained hyperprolactinaemia, with a mean prolactin level of $2933 \mathrm{mU} / \mathrm{l}$ (range 1125-6000), and pituitary imaging (either computed tomography (CT) or magnetic resonance imaging (MRI)) in 27 patients, which showed a pituitary microadenoma (diameter $10 \mathrm{~mm}$ or less) in 18 patients. There was no significant change in the prolactin normal range $(<600 \mathrm{mU} / \mathrm{l})$ during the period of study.

At time of referral, prior to dopamine agonist treatment, 31 patients $(96.8 \%)$ had menstrual disturbance, galactorrhoea was present in $15(46.9 \%)$ and infertility a presenting problem in 15 (46.9\%). Bromocriptine intolerance (such that patients could not continue to take a dopamine agonist at the dose necessary for restoration of menses and ovulation and/or normalisation of prolactin) was the indication for surgery in the majority of cases (ten patients (31\%)), and three of these patients were also intolerant of cabergoline. Six patients (18\%) were resistant (failure to suppress prolactin to greater than half the starting level, despite escalating doses of dopamine agonist) to bromocriptine, and one was also resistant to cabergoline. Four patients $(12 \%)$ were resistant and intolerant of bromocriptine. Two patients were from countries where dopamine agonists were not easily available, and in others, where specified, the indication was patient preference. Nineteen patients $(59 \%)$ received preoperative treatment with dopamine agonists, and this was bromocriptine in the majority of cases, although one patient also received quinagolide and four also tried cabergoline. The duration of treatment varied from 10 days to 10 years, but was intermittent in many patients.

The data were analysed using the chi-squared test and Wilcoxon's rank sum test, using $P<0.05$ as significant.

\section{Results}

\section{Surgical outcome}

Surgical cure, following selective adenectomy, defined as a post-operative prolactin in the normal biochemical range in the absence of dopamine agonist therapy, was achieved in $25(78 \%)$ patients (Table 1$)$. The mean
Table 1 Surgical outcome.

\begin{tabular}{lcc}
\hline & Number of patients & Mean prolactin $(\mathrm{mU} / \mathrm{l})$ \\
\hline Remission & $25(78 \%)$ & 253 \\
Uncured & $7(22 \%)$ & 1897 \\
Recurrence & $1(4 \%)$ & 3885 \\
\hline
\end{tabular}

post-operative prolactin level was $253 \mathrm{mU} / \mathrm{l}$ (range, from not detected to $470 \mathrm{mU} / \mathrm{l}$ ) in the cured group and $1897 \mathrm{mU} / \mathrm{l}$ (range 1772-1850) in the uncured group.

Surgery was well-tolerated, and was complicated by diabetes insipidus that was temporary in two patients and permanent in five (15.6\%).

One patient with a normal post-operative prolactin (346 mU/l) had evidence of persistent hyperprolactinaemia (prolactin $817 \mathrm{mU} / \mathrm{l}$ ) and galactorrhoea at 3 months. She underwent a second operation that was curative.

One patient developed recurrent hyperprolactinaemia at 12 years with no evidence of tumour on imaging.

Two patients had a suboptimal cortisol response during an insulin tolerance test post-operatively, but all other patients had normal adrenocorticotrophin $(\mathrm{ACTH})$ reserve as judged by an insulin tolerance test in 26 patients, short synacthen test in 2 patients, and random cortisol $>600 \mathrm{nmol} / \mathrm{l}$ in 1 patient. There was no information available on one patient.

Two patients were thyrotrophin (TSH) deficient postoperatively and one developed primary hypothyroidism, but the remainder had normal thyrotroph function. Out of 21 patients tested for growth hormone reserve with an insulin tolerance test, $6(28.6 \%)$ were growth hormone deficient. Nine patients had luteinising hormone (LH)-releasing hormone tests, and 17 patients had LH in the normal range. Two had day 21 progesterone levels checked and these were both compatible with ovulation. There was no information on LH reserve in three patients, although one of these was documented to be having regular periods. Only one patient (cured) had post-operative LH deficiency.

Twenty-one of 28 patients $(75 \%)$ were documented to be menstruating regularly, two were taking the oral contraceptive pill and two had other causes for amenorrhoea (hysterectomy, absent vagina). Ten of these patients were known to have had successful pregnancies following surgery. Three patients were not menstruating regularly and these were all uncured.

\section{Characteristics of patients who were not cured by surgery}

Seven patients (22\%) were not cured by surgery (Table 2), although one of these patients was 'clinically' cured with restoration of menses, ovulation and fertility. There was no significant difference between pre-operative prolactin in the cured $(2717 \mathrm{mU} / \mathrm{l})$ and uncured (3704 mU/l) groups. 
Table 2 Characteristics of patients cured and not cured by surgery.

\begin{tabular}{lll}
\hline & Cured & Uncured \\
\hline Number of patients & $25 / 32(78 \%)$ & $7 / 32(22 \%)$ \\
Pre-operative prolactin (mU/I) & 2717 & 3704 \\
Pre-operative bromocriptine & $14 / 25(56 \%)$ & $5 / 7(71 \%)$ \\
Tumour detected on imaging & $14 / 25(56 \%)$ & $4 / 7(57 \%)$ \\
Tumour detected by surgeon & $22 / 22(100 \%)$ & $6 / 6(100 \%)$ \\
Confirmatory histology & $14 / 19(74 \%)$ & $1 / 5(20 \%)$ \\
\hline
\end{tabular}

There was no significant difference in terms of preoperative bromocriptine treatment when the uncured group were compared with the cured patients: 4 patients $(80 \%)$ had received pre-operative bromocriptine for between 3 weeks and 6 months, whereas 14 $(73.7 \%)$ of the cured patients had received bromocriptine prior to surgery, for a period of 10 days up to 10 years $(P>0.05)$.

Whether a microadenoma was demonstrated on either CT or MRI, or whether a tumour was detected by the surgeon, was not significantly associated with outcome $(P>0.05)$. Neither resistance to treatment with bromocriptine nor intolerance was predictive of lack of cure.

Histology confirmed the presence of pituitary adenoma in 14/19 of the cured patients. Immunostaining for prolactin was confirmatory in 13 cases and unavailable in 1. In contrast, pituitary adenoma was only demonstrated histologically in one of five uncured patients with available histological records, and immunostaining was not performed at this time. Microscopic examination of the other cases showed normal pituitary gland only.

\section{Estimated costs}

A comparison of costs of trans-sphenoidal surgery or drug treatment is shown in Table 3. This considers a hypothetical patient undergoing surgery and cure with no complications, and followed up for 10 years, and compares this with the cost of a patient receiving treatment with cabergoline $1 \mathrm{mg}$ /week for 10 years.

\section{Discussion}

Our data show that trans-sphenoidal surgery for microprolactinoma is associated with a $78 \%$ cure rate and recurrence rate of only $1 / 25$ cases $(4 \%)$. This cure rate is comparable with other series (9-13) and the recurrence rate of only $4 \%$ is lower than that often quoted: $12 \%$ (14), $17.4 \%$ (3), 21\% (12) and 50\% (9). There were few complications of surgery: five patients developed permanent diabetes insipidus (15.6\%), two developed TSH deficiency, and two had reduced ACTH reserve on insulin tolerance test post-operatively. The commonest post-operative hormonal deficiency was growth hormone in $28.6 \%$ of the patients. There are no comparable data on growth hormone deficiency following trans-sphenoidal surgery for microprolactinoma in other surgical series, but it is clearly important to counsel patients about this possibility prior to surgery. It is not clear why the incidence of diabetes insipidus was higher than that found in patients operated on for acromegaly, by the same surgeon

Table 3 Relative costs of surgery and medical treatment.

\begin{tabular}{lll}
\hline & Surgery & Drug treatment \\
\hline New patient appointment & $£ 77$ & $£ 77$ \\
MRI pituitary & $£ 105$ & $£ 105$ \\
Follow-up appointments in first year & & $3 \times £ 39=£ 117$ \\
Pituitary function tests & & $£ 60$ \\
Inpatient stay (includes pituitary function tests and visual fields) & $£ 2580$ & \\
Trans-sphenoidal surgery & $£ 1362$ & \\
Antibiotics & $£ 61$ & \\
Post-operative assessment (includes pituitary function tests) & $£ 165$ & \\
Post-operative MRI & $£ 105$ & \\
Post-operative visual fields & $£ 70$ & \\
Annual prolactin for: & & \\
5 years & $£ 5$ & $£ 5$ \\
10 years & $£ 10$ & $£ 10$ \\
Outpatient appointments for: & $£ 39$ & $£ 39$ \\
5 years & $£ 195$ & $£ 195$ \\
10 years & $£ 390$ & $£ 390$ \\
Cabergoline 1 mg/week for: & & $12 \times £ 31.46=$ \\
5 years & & $£ 1887.60$ \\
10 years & & $£ 3775.20$ \\
Total & & \\
5 years & & \\
10 years & $£ 4725$ & $£ 2446.60$ \\
\end{tabular}


at the same institution over the same time period $(5.8 \%)$.

A summary of the results from 31 series shows that $872 / 1224(71.2 \%)$ of patients with microprolactinomas had normal prolactin levels after surgery, although the range varied from 38 to $91 \%$ (3). This series reports a recurrence rate of $82 / 471(17.4 \%)$, so reducing the long-term normalisation of prolactin levels with surgery from this review to $52.8 \%$. Others have reported higher recurrence rates of up to $50 \%$ (9), although a further assessment of 57 patients with microprolactinomas showed that although 25 women relapsed with hyperprolactinaemia during a mean follow-up of 3 years, many of the women were asymptomatic, making a clinical remission rate of $75 \%, 10-20$ years after initial successful trans-sphenoidal surgery for microprolactinomas (15). A 10 year follow-up study of 61 patients who underwent trans-sphenoidal surgery for microprolactinoma demonstrated a cure rate of $73 \%$ in terms of hyperprolactinaemia, with a recurrence rate of $12 \%$ (14). This study, like the series reported by Serri et al. (15), showed that recurrences continued to occur up to 10 years post-operatively. It may be that our recurrence rate will rise as the duration of follow-up increases.

A comparison of dopamine agonist treatment showed that 5 of the 7 uncured had received prior unsuccessful treatment with dopamine agonists (intolerance in 4 of $5)$, compared with 14 of the patients who were cured $(P>0.05)$. These data do not confirm the suggestion that a lack of response to dopamine agonists or poor tolerability is characteristic of an active form of tumour and therefore resistance to surgical cure (16). It is difficult to judge duration of treatment and compliance in retrospective analyses. Many patients had taken bromocriptine intermittently, and true bromocriptine resistance is often difficult to differentiate from intolerance. This may partly explain the difference from the work of Soule et al. (16), but it is also important to note that their study included patients with micro- and macroprolactinomas, and that all 11 patients with microprolactinomas had received dopamine agonist treatment pre-operatively. Since it is the patient with a tumour resistant to dopamine agonist treatment, or who is intolerant of such treatment, who is more likely to be referred for trans-sphenoidal surgery, it is important that our data demonstrate that they do not necessarily fall into a group with an adverse prognosis.

It is perhaps surprising that the identification of a microadenoma on pituitary imaging was not significantly associated with cure following trans-sphenoidal surgery. Many feel that an essential pre-requisite to surgery is the finding of a definite lesion on pituitary imaging (10). Trans-sphenoidal surgery in patients with no demonstrable tumour carried a poor success rate of $54 \%$ in one series (17). However, approximately $10 \%$ of asymptomatic subjects harbour pituitary incidentalomas detectable either at autopsy or on imaging $(18,19)$, and therefore scans may produce false positive results (17). Thus based on our data, where tumours were only identified in $18 / 27$ patients imaged, the lack of a demonstrable microadenoma on imaging in a patient with a significantly elevated prolactin level where other causes of hyperprolactinaemia have been excluded, should not alter the decision as to whether to ask an experienced surgeon to operate. In these cases the surgeon is often able to identify a clear microadenoma despite the lack of positive imaging. It is interesting to compare imaging and outcome between the two decades. During the first 10 years, 15/20 patients had CT scans performed. (The remainder had skull X-rays and metrimazole cisternograms.) Only just over half $(8 / 15(53.3 \%))$ of the scans demonstrated the tumour. During the second decade, all patients had CT or MRI scanning and 10/12 (83\%) of these investigations were 'positive', showing the tumour. Interestingly, there was no significant difference in cure rate between the two decades ( 80 and $75 \%, P>0.05)$.

The estimates for cost that we have made take an uncomplicated patient receiving either dopamine agonist treatment or trans-sphenoidal surgery. Clearly lack of cure or hypopituitarism including symptomatic growth hormone deficiency would add significant costs to surgical treatment, while increased dosage requirements or a couple of pregnancies requiring monitoring of visual fields and possibly a post-partum MRI, would increase the cost of medical treatment. We have not compared oestrogen treatment alone as fertility is an important issue for many of these patients, but this would usually be a cheaper option. There is also the possibility of remission in one-third of patients with hyperprolactinaemia following pregnancy (20). It is interesting to note that, despite these caveats, the two modalities of treatment do not differ significantly in terms of cost over 10 years.

This study shows that trans-sphenoidal surgery in the dopamine agonist era, in the hands of an experienced pituitary surgeon, is associated with a high cure rate with few post-operative complications and a low recurrence rate. These data compare favourably with the outcome following dopamine agonist treatment, with the advantage that surgery obviates the need to continue drug treatment which may be associated with unpleasant side-effects and may lack adequate safety data during pregnancy. Clearly we do not advocate trans-sphenoidal surgery for everyone with a microprolactinoma. These data obviously cannot be extrapolated to all centres treating patients with microprolactinomas, as dopamine agonists work well in most cases when prescribed by anyone, in contrast to surgery. However, where there is access to an expert in trans-sphenoidal surgery, and where dopamine agonist tolerance/resistance is a problem, then the option of trans-sphenoidal surgery should be strongly considered. Perhaps the time has come for a randomised trial, or at least the consideration of surgery earlier in the management strategy. 


\section{References}

1 Thorner MO, McNeilly AS, Hagan C \& Besser GM. Long-term treatment of galactorrhoea and hypogonadism with bromocriptine. British Medical Journal 19742 419-422.

2 Bevan JS, Webster J, Burke CW \& Scanlon MF. Dopamine agonists and pituitary tumour shrinkage. Endocrine Reviews 1992131 220-240.

3 Molitch ME. Pathologic hyperprolactinaemia. Endocrinology and Metabolism Clinics of North America 199221 877-901.

4 Webster J, Piscitelli G, Polli A, D’Alberton A, Falsetti L, Ferrari C et al. The efficacy and tolerability of long-term cabergoline therapy in hyperprolactinaemic disorders: an open uncontrolled, multicentre study. Clinical Endocrinology 199339 323-329.

5 Webster J, Piscitelli G, Polli A, Ferrari CI, Ismail I, Scanlon MF for the Cabergoline Comparative Study Group. A comparison of cabergoline and bromocriptine in the treatment of hyperprolactinaemic amenorrhoea. New England Journal of Medicine 1994331 904-909.

6 Ferrari C, Paracchi A, Mattei AM, de Vincentis S, D'Alberton A \& Crosignani P. Cabergoline in the long-term therapy of hyperprolactinaemic disorders. Acta Endocrinologica 1992126 489-494.

7 Colao A, Di Sarno A, Sarnacchiaro F, Ferone D, Di Renzo G, Merola B et al. Prolactinomas resistant to standard dopamine agonists respond to chronic cabergoline treatment. Journal of Clinical Endocrinology and Metabolism 199782 876-883.

8 Molitch M, Thorner MO \& Wilson C. Management of prolactinomas. Therapeutic controversy. Journal of Clinical Endocrinology and Metabolism 199782 996-1000.

9 Serri O, Rasio E, Beauregard H, Hardy J \& Somma M. Recurrence of hyperprolactinaemia after selective adenomectomy in women with prolactinoma. New England Journal of Medicine 1983309 280-283.

10 Scanlon MF, Peters JR, Thomas JP, Richards SH, Morton WH, Howell S et al. Management of selected patients with hyperprolactinaemia by partial hypophysectomy. British Medical Journal $19852911547-1550$.

11 Thomson JA, Teasdale GM, Gordon D, McCruden DC \& Davies DL. Treatment of presumed prolactinoma by trans-sphenoidal operation: early and late results. British Medical Journal 1985291 1550-1553.

12 Rodman EF, Molitch ME, Kalmon PD, Biller BJ \& Reichlin S. Longterm follow-up of trans-sphenoidal selective adenomectomy for prolactinoma. Journal of the American Medical Association 1984 252 921-924.

13 Scamoni C, Balzarini C, Crivelli G \& Dorizzi A. Treatment and long-term follow-up results of prolactin secreting pituitary adenomas. Journal of Neurosurgical Sciences 199135 9-16.

14 Thomson JA, Davies DL, McClaren E \& Teasdale GM. Ten year follow-up of microprolactinoma treated by trans-sphenoidal surgery. British Medical Journal 1994309 1409-1410.

15 Serri O, Hardy J \& Massoud F. Relapse of hyperprolactinaemia revisited. New England Journal of Medicine 19933291357.

16 Soule SG, Farhi J, Conway GS, Jacobs HS \& Powell M. The outcome of hypophysectomy for prolactinomas in the era of dopamine agonist therapy. Clinical Endocrinology 199644 711-716.

17 Webster J, Page MD, Bevan JS, Richards SH, Douglas-Jones AG \& Scanlon MF. Low recurrence rate after partial hypophysectomy for prolactinoma: the predictive value of dynamic prolactin function tests. Clinical Endocrinology 199236 35-44.

18 Molitch ME \& Russell EJ. The pituitary 'incidentaloma'. Annals of Internal Medicine 1990112 925-931.

19 Hall WA, Luciano MG, Doppman JL, Patronas NJ \& Oldfield EH. Pituitary magnetic resonance imaging in normal human volunteers: occult adenomas in the general population. Annals of Internal Medicine 1994120 817-820.

20 Jeffcoate WJ, Pund N, Sturrock NDC \& Lambourne J. Longterm follow-up of patients with hyperprolactinaemia. Clinical Endocrinology 199645 299-303.

Received 13 October 1998

Accepted 13 October 1998 